\title{
Resolution of vocal fold polyps with conservative treatment
}

\author{
Commented by: Glaucya Madazio ${ }^{1}$, Felipe Moreti $^{2}$
}

Nakagawa H, Miyamoto M, Kusuyama T, Mori Y, Fukuda H. Resolution of vocal fold polyps with conservative treatment. J Voice. 2012;26(3):e107-10.

Vocal fold polyps are relatively common benign laryngeal lesions that produce persistent hoarseness, usually treated by surgical removal. The lesion can be reddish, whitish or translucent and the image is of an elevated lesion located on the free edge of the vocal fold at the junction of the anterior and middle third. It is believed that vocal abuse is the base of its development by promoting rupture of vessels in the superficial layer of the lamina propria and consequently producing hematoma. This process follows with edema and inflammatory cellular infiltration that produces a new matrix. The presence of the lesion mechanically constrains the approximation of the vocal folds during the closed phase of the glottal cycle and causes an increase of vocal effort and rugosity. The increase of subglottic pressure and intralaryngeal muscular hyperfunction are additional aspects to the phonotrauma that promotes the maintenance of the lesions.

Although surgery is the preferably adopted treatment, some surgeons have noticed that a percentage of polyps withdraw. However, this rate is small and the characteristics of such lesions are not known. Regarding voice treatment, there is no consensus about the recommendation of voice therapy.

The purpose of the study is to identify the frequency of spontaneous resolution of polyps and the characteristics of these lesions in order to establish a better definition for treatment strategy. A review of medical records of 644 patients in Tokyo Voice Center, from January 2001 to January 2008 was carried out. Vocal fold polyps were defined as persistent unilateral lesion, located in the middle of the musculo-membranous portion of the vocal folds, with an atypical, hemorrhagic, fibrotic and translucent appearance. All patients that did not show up for a follow-up appointment and that had the diagnosis of nodules, Reinke's edema, leukoplakia and granuloma were excluded. Every patient had a videostroboscopic examination to confirm the diagnosis. The polyps were subdivided into three categories according to the size: small (pinpoint base), medium (base greater than a pinpoint, but smaller than a third of the vocal fold) and large (base greater than a third of the vocal fold length). At this center, polyps are usually treated by laryngeal microsurgery just like they are at many other clinics and centers. Conservative treatment is indicated for patients that refused undergoing surgery, or who have any risk for the

(1) Centro de Estudos da Voz - CEV - São Paulo (SP), Brazil.

(2) Centro de Estudos da Voz - CEV - São Paulo (SP), Brazil; Department of Speech-Language Pathology and Audiology, Universidade Federal de São Paulo - UNIFESP - São Paulo (SP), Brasil.

Correspondence address: Glaucya Madazio. R. Machado Bittencourt, 361/1001, São Paulo (SP), Brazil, CEP: 04044-905. E-mail: glaumadazio@uol.com.br surgical procedure or general anesthesia, or even for those who have their lesions improved while waiting for operation. When the selected treatment is conservative, voice therapy and/or medication (steroids) are administered according to the patient's preferences. The voice therapy protocol is determined by the speech pathologist and, in general, it includes counseling, vocal hygiene and breath support. Voice therapy was administered at the same institution with intervals of one to four weeks between sessions.

Out of the 644 patients diagnosed with polyp during the period mentioned previously, 132 received conservative treatment. Out of the remaining 433 patients that were submitted to surgery, 79 of them did not show up to the follow-up appointment. The mean age of patients submitted to conservative treatment was 47.8 years (range of 20 to 83 years), 79 of them were female $(56.1 \%)$ and 58 were male $(43.9 \%)$. At the evaluation consultation, patients had been having hoarseness for a mean period of 7.3 months with a range of 0 days (polyp was found by chance at the medical appointment) to 20 years. On videostroboscopic examination, 26 polyps were large, 58 medium and 46 small in size.

Of the 132 patients that underwent conservative treatment, $55(41.7 \%)$ had complete resolution of the lesions after a mean period of 5.1 months of follow-up and 29 (21.9\%) had the lesion diminished in size and were satisfied with the results as far as vocal quality was concerned, after a mean of 4.1 months of follow-up. The remaining $48(36.4 \%)$ patients showed no changes on the lesion appearance, of which 17 were satisfied with their voices regardless of the presence of the polyp and 31 of them, including the 14 that were recommended surgery, quit treatment after 3.2 months. Comparing those patients who had complete resolution of the lesion to the ones that did not show resolution or reduction of the polyp, the first group has a greater proportion of women (58.2\% versus $41.7 \%$ ), small lesions (45.4\% versus $22.9 \%$ ) and shorter duration of symptoms (2.8 weeks versus 13.9 weeks). None of the patients that had complete resolution of the polyp presented with recurrence of the lesion.

Voice therapy was administered to 38 patients, 24 of them also were prescribed with medication. $10(26.3 \%)$ patients had complete remission of the lesion with voice therapy and $8(21.1 \%)$ had some reduction of the lesion. On the other hand, of the 94 patients that did not receive voice therapy, 45 (47.9\%) had complete resolution and $20(21.3 \%)$ had their lesions reduced in size.

Mean age of surgically treated group was 46.7 years (from 8 to 81 years), 234 out of the 433 participants were women (54\%) and 199 were men (46\%). Patients presented hoarse- 
ness for a mean of 9.8 months (ranging from 0 to 15 years). Regarding the size of the lesion, 180 polyps were large, 206 medium and 27 small. The length between the first appointment and the surgery was 1.4 months. Follow-up mean duration after surgery was 4.9 months. The majority of the individuals had vocal improvement, however 26 (6\%) experienced lesion recurrence. In summary, the findings of the present study suggest that approximately $10 \%$ of the polyps can be resolved with conservative treatment. Although the standard treatment for these lesions is surgery, it is important to avoid unnecessary procedures. If the polyp is relatively small or has a recent onset, patients should be followed-up closely for some months with the expectation of resolution, in cases that there is not vocal demand that requires quick recovery. It is interesting to note that when the patients that experienced complete remission are compared to those that did not have resolution or reduction of the lesion, the former group is composed of mainly women with small lesions and recent symptoms. A possible reason for the women to have shown better result is that these patients cooperated more with the controlling of vocal abuse, consequently reducing subglottic pressure and less muscle activation. It is thought that the same process that is involved in the development of polyps can lead to a remo- deling of the tissue and therefore, promoting its reabsorption. Another possibility is that the ongoing phonotrauma causes a reduction of the polyp's base and can eventually promote its rupture and removal.

The present retrospective study cannot demonstrate that voice therapy is an effective treatment, since there was not a controlled design. Same thing can be stated about the use of steroids for treating these lesions. The retrospective nature of this study introduces biases and only further researches can allow the verification of the real effectiveness of voice therapy. If on the one hand, conservative treatment showed results with a mean of 5.1 months, on the other hand the benefit of a very fast recovery with surgery should be taken in consideration. Even though surgery is a common, fast and controlled procedure, there are costs and risks associated with general anesthesia, hospitalization, absolute vocal rest, the surgery itself and vocal fold scarring.

Therefore, voice therapy can be considered the first treatment option for polyps, especially for women with small lesions and translucent appearance, and objective signs of muscle tension and complete glottal closure. These characteristics can aid the otorhinolaryngologist to guide the patients that will benefit from voice rehabilitation. 